Original

\title{
Translation and validation of the Arabic version of the Geriatric Oral Health Assessment Index (GOHAI)
}

\author{
Shaher Daradkeh ${ }^{1)}$ and Yousef S. Khader ${ }^{2)}$ \\ ${ }^{1)}$ Ministry of Health, Irbid, Jordan \\ ${ }^{2)}$ Health and Medical Sciences Sector, Higher Council for Science and Technology, \\ Department of Community Medicine, Public Health and Family Medicine, Faculty of Medicine, \\ Jordan University of Science and Technology, Irbid, Jordan
}

(Received 16 June and accepted 22 October 2008)

\begin{abstract}
Our aim was to translate the original English version of the Geriatric Oral Health Assessment Index (GOHAI) into Arabic and assess its validity and reliability for use among people in North Jordan. After translation into Arabic and back-translation to check the translation quality, a total of 288 participants completed the Arabic version of the GOHAI questionnaire. Individual GOHAI items were recoded and summed as originally recommended. The questionnaire sought information about sociodemographic characteristics and self-reported perception of general and oral health. Clinical examination included assessment of periodontal status, and number of decayed teeth, missing teeth, filled teeth and crowned teeth. Reliability, internal consistency, and concurrent, convergent and discriminant validity of GOHAI scores were examined. Mean GOHAI score was 40.9 ( $S D=10.6$, range: 12 to 60). Cronbach's alpha for the GOHAI score was 0.88 , indicating a high degree of internal consistency and homogeneity between the GOHAI items. The test-retest correlation coefficient for add-GOHAI scores was 0.72 , indicating good stability. Add-GOHAI scores increased with poorer perceived general and oral health. Convergent validity, construct validity and discriminant validity of the GOHAI were demonstrated. The Arabic translation of the GOHAI

Correspondence to Dr. Yousef S. Khader, Health and Medical Sciences Sector, Higher Council for Science and Technology, Department of Community Medicine, Public Health and Family Medicine, Faculty of Medicine, Jordan University of Science and Technology, Irbid 22110, Jordan

Tel: $+962-795435025$

Fax: +962-2-7201064

E-mail: yousef.k@excite.com
\end{abstract}

demonstrated acceptable validity and reliability when used for people in North Jordan. It could therefore be used as a valuable instrument for measuring oral health-related quality of life for people in this region. (J. Oral Sci. 50, 453-459, 2008)

Keywords: validity; reliability; oral health; quality of life; Arabic; Jordan.

\section{Introduction}

Measures of oral health-related quality of life (OHQoL) are essential for epidemiological and clinical studies in order to provide accurate data for health promotion, disease prevention programs and allocation of health resources (1). Other uses of such measures were described by Fitzpatrick et al. (1) and Slade and Spencer (2). Most of the OHQoL instruments (2-6) that have been shown to have adequate validity and reliability are based on three main dimensions: physical symptoms, perception of well-being and functional capacity. Among the most commonly used instruments is the Geriatric Oral Health Assessment Index (GOHAI) (3). This has been validated and widely used in North America. Its internal consistency is satisfactory and its concurrent and construct validity have been confirmed (7). Swedish (8), Malay (9), Chinese (10) and French (11) versions have shown acceptable reliability and validity. However, none of the indicators of OHQoL have been validated in Arabic for use among people in North Jordan.

It is important that an adopted instrument should be culturally relevant and valid for the local population while demonstrating acceptable psychometric properties (1214). It is therefore essential to carry out a rigorous translation and validation process of the instrument when used in 
another population with a different culture. Therefore, this study was conducted to translate the original English version of the GOHAI into Arabic and validate it to evaluate the impact of oral disease on the quality of life of people in north Jordan

\section{Study population}

\section{Methods}

A total of 300 consecutive people who had attended North Badia Comprehensive Health Center for any reason over a period of two months were invited to participate in this study. After providing verbal consent to participate, $290(96.7 \%)$ people were clinically examined. Two subjects were excluded from the study because their questionnaire answers were incomplete, leaving 288 to be included in the analysis.

\section{Translation and scoring of the GOHAI}

The GOHAI was translated into Arabic by two dentists who were fluent in both English and Arabic. The Arabic draft was then back translated into English by another two people fluent in both Arabic and English. The backtranslated version was compared with the original English version to verify that the questions were properly translated. All of the back-translated items were worded similarly to the original ones and were comparable in their meaning. The Arabic draft was then discussed by three dentists who found that it would aid understanding to rephrase the questions in the form of simple statements instead of question format without changing their meaning. For example, GOHAI item 1 "How often do you limit the kinds or amounts of food you eat because of problems with your teeth or dentures?" was restated as: "Because of problems with my teeth or dentures, I limit the kinds or amounts of food I eat".

Individual GOHAI items were recoded and summed as originally recommended. Two summary scores were calculated. Add-GOHAI was calculated as the sum of values of responses to the 12 items for each participant with the resulting score ranging from 12-60. Simple count score (SC-GOHAI) was calculated by counting the number of GOHAI items with responses "sometimes", "often", or "always". Less than 5\% of values were missing, and these were replaced with the median of all values in the series.

\section{Questionnaire}

In addition to the 12 items of the GOHAI, the questionnaire included socio-demographic characteristics such as age, sex, educational level, marital status, employment, and income. Subjects were also asked about their perception of their general and oral health, whether they were satisfied with their dental condition, their assessment of their need for dental treatment, pain or discomfort due to temporomandibular joint (TMJ) disorders, burning mouth sensation, sensation of TMJ clicking, and bad oral habits such as biting objects. Because of the high illiteracy rate in North Badia, the questionnaire was completed through personal interview.

\section{Clinical examination}

The researchers assessed periodontal status and number of decayed teeth, missing teeth, filled teeth, and crowned teeth. Sterile dental mirrors and standardized periodontal probes were used to measure probing pocket depth (PPD) and clinical attachment level (CAL). PPD was measured from the gingival margin to the bottom of the crevice to the nearest millimeter ( $\mathrm{mm}$ ). If the cement-enamel junction (CEJ) was exposed, CAL was measured by reading off the distance from the CEJ or the margin of fixed restoration to the base of the pocket. In other cases it was measured indirectly by subtracting the distance from the gingival margin to the CEJ from the pocket depth, with the tip of the probe used to feel for the CEJ level. In both cases, CAL was measured to the nearest $\mathrm{mm}$.

PPD and CAL were measured at six sites (mesio-facial, mid-facial, disto-facial, mesio-lingual, mid-lingual, and disto-lingual) per tooth for all teeth, excluding third molars. The number of decayed teeth, filled teeth, and missing teeth for each participant were recorded. Periodontitis was defined as presence of four or more teeth with PPD $\geq 4 \mathrm{~mm}$ and $\mathrm{CAL} \geq 3 \mathrm{~mm}$ at one site or more.

\section{Reliability}

Cronbach's alpha was calculated to assess the degree of internal consistency and homogeneity between items (15). Pearson's correlation coefficient was used to measure item-scale correlation to assess the correlation between the individual items and their scale score. To assess test-retest reliability, 30 participants repeated the GOHAI one week after the questionnaire was first administered. Test-retest reliability was measured using Pearson's correlation coefficient for individual items and for the overall GOHAI score.

\section{Validity}

Concurrent validity was investigated by examining the degree to which the GOHAI scores were related to the scores of four self-reported items: general health, oral health, need for dental care, and satisfaction with oral health status. We assessed the ability of the GOHAI to distinguish between groups of people with different responses to these self-reported items, which it should 
theoretically be able to do. Convergent validity was evaluated by examining the association between GOHAI scores and objective assessment of oral and dental status (number of missing teeth, number of decayed teeth, and the presence or absence of periodontal disease) and selfreported symptoms with hypothesized effects on oral health-related quality of life: TMJ pain, burning mouth sensation, and bad breath). It was predicted that subjects who did not have one of such symptoms would have better oral health-related quality of life and thus higher GOHAI scores compared with subjects who had that symptom $(11,16)$. Discriminant validity was evaluated by examining the association between GOHAI scores and self-reported bad oral habits that were hypothesized not to be notably associated with oral health-related quality of life (16) and thus to have no effect on GOHAI scores.

\section{Statistical analysis}

Statistical Package for Social Sciences (SPSS, version 11.5) software was used to analyze data. Frequency distributions were produced. Means and standard deviations of the dependent variables (Add-GOHAI and SC-GOHAI scores) were estimated and compared among different groups of the study population using a $t$-test or one-way ANOVA as appropriate. Pearson's correlation coefficient was used to measure item-scale correlations. Responses to the 12-item questionnaire were subjected to factor analysis using squared multiple correlations as prior communality estimates. The principal components method of factor extraction and varimax method of factor rotation were used in factor analysis. A $P$-value of less than 0.05 was considered statistically significant.

\section{Results \\ Participants' characteristics}

A total of 288 participants (155 men and 133 women) agreed to participate and completed the GOHAI questionnaire. Their socio-demographic characteristics are shown in Table 1 . Their mean age $( \pm \mathrm{SD})$ was $33.4( \pm$ 13.2) years. A total of $134(48.6 \%)$ respondents were 30 years of age or younger and $184(63.9 \%)$ were married. Monthly income was $160 \mathrm{JD}$ or less ( $1 \$=0.7 \mathrm{JD})$ for 106 $(50.7 \%)$ respondents, and only $44(15.3 \%)$ had attained more than high school education. More than two thirds of participants $(70.7 \%)$ reported that they visited the dentist only when they had pain and $118(42.6 \%)$ reported that they brushed their teeth once or more per day.

\section{Self-perceived general and oral health}

Table 2 shows the distribution of respondents according to different self-reported ratings of health. Seventy-one
Table 1 Socio-demographic and important characteristics of participants

\begin{tabular}{|c|c|c|}
\hline Variable & $\mathrm{n}$ & $(\%)$ \\
\hline \multicolumn{3}{|l|}{ Gender } \\
\hline Male & 155 & (53.8) \\
\hline Female & 133 & $(46.2)$ \\
\hline \multicolumn{3}{|l|}{ Age (year) } \\
\hline$\leq 30$ & 134 & $(48.6)$ \\
\hline$>30$ & 142 & $(51.4)$ \\
\hline \multicolumn{3}{|l|}{ Marital status } \\
\hline Married & 184 & $(63.9)$ \\
\hline Not married & 104 & (36.1) \\
\hline \multicolumn{3}{|l|}{ Educational level } \\
\hline Illiterate & 80 & $(27.8)$ \\
\hline High school or less & 164 & $(56.9)$ \\
\hline More than high school & 44 & $(15.3)$ \\
\hline \multicolumn{3}{|l|}{ Income (JD) } \\
\hline$\leq 160$ & 106 & $(50.7)$ \\
\hline$>160$ & 103 & $(49.3)$ \\
\hline \multicolumn{3}{|l|}{ Smoking habit } \\
\hline Smoker & 89 & $(30.9)$ \\
\hline Non-smoker & 199 & $(69.1)$ \\
\hline \multicolumn{3}{|l|}{ Visit to the dentist } \\
\hline Regular & 39 & $(13.6)$ \\
\hline Not regular & 45 & $(15.7)$ \\
\hline On pain & 203 & $(70.7)$ \\
\hline \multicolumn{3}{|l|}{ Tooth brushing } \\
\hline$\geq 1 /$ day & 118 & $(42.6)$ \\
\hline Not regular & 109 & $(39.4)$ \\
\hline Never & 50 & (18.1) \\
\hline
\end{tabular}

(24.8\%) respondents reported that they had excellent general health and 95 (33.2\%) reported that they had very good general health. Only $11.5 \%$ of the participants reported that they had excellent oral health and $18.1 \%$ perceived their oral health as being very good. Three quarters of the subjects perceived themselves as needing dental treatment. Only $36.9 \%$ of participants were satisfied with their oral health status.

\section{Oral health}

Oral health examination showed that $65.6 \%$ of subjects had at least one decayed tooth, $61.8 \%$ had at least one missing tooth, $49.0 \%$ had one or more filled teeth, and $16.3 \%$ had one or more crowned teeth. Periodontal disease was present in $33.3 \%$ of the subjects.

\section{Acceptability of and responses to the Arabic version of the GOHAI}

The frequency of missing responses for GOHAI items ranged from $0.1 \%$ to $3.8 \%$.Using the "add scoring" method, only one subject had a minimum score of 12 , indicating the greatest possible impact from oral conditions, and 9 
Table 2 Distribution of participants according to self-reported health-related ratings

\begin{tabular}{|c|c|c|}
\hline \multirow[t]{2}{*}{ Variable } & \multicolumn{2}{|c|}{ Frequency } \\
\hline & & $(\%)$ \\
\hline \multicolumn{3}{|c|}{ Self-reported general health } \\
\hline Excellent & 71 & $(24.8)$ \\
\hline Very good & 95 & $(33.2)$ \\
\hline Good & 78 & $(27.3)$ \\
\hline Moderate & 32 & $(11.2)$ \\
\hline Bad & 10 & $(3.5)$ \\
\hline \multicolumn{3}{|c|}{ Self-reported oral health } \\
\hline Excellent & 33 & $(11.5)$ \\
\hline Very good & 52 & $(18.1)$ \\
\hline Good & 77 & $(26.8)$ \\
\hline Moderate & 81 & $(28.2)$ \\
\hline Bad & 44 & $(15.3)$ \\
\hline \multicolumn{3}{|c|}{ Self-perceived need for dental care } \\
\hline Yes & 216 & $(75.0)$ \\
\hline No & 72 & $(25.0)$ \\
\hline \multicolumn{3}{|c|}{ Self-reported satisfaction with oral health } \\
\hline Yes & 106 & $(36.9)$ \\
\hline No & 181 & $(63.1)$ \\
\hline \multicolumn{3}{|c|}{ Self-reported TMJ pain during last month } \\
\hline Yes & 106 & $(36.8)$ \\
\hline No & 182 & $(63.2)$ \\
\hline \multicolumn{3}{|c|}{ Self-reported TMJ clicking during last month } \\
\hline Yes & 68 & $(23.7)$ \\
\hline No & 219 & $(76.3)$ \\
\hline \multicolumn{3}{|c|}{ Self-reported bad breath } \\
\hline Often & 35 & $(12.2)$ \\
\hline Sometimes & 128 & $(44.6)$ \\
\hline Rarely & 48 & $(16.7)$ \\
\hline Never & 76 & $(26.5)$ \\
\hline \multicolumn{3}{|c|}{ Self-reported burning mouth sensation } \\
\hline Yes & 63 & $(22.2)$ \\
\hline No & 221 & $(77.8)$ \\
\hline \multicolumn{3}{|c|}{ Self-reported bad oral habits } \\
\hline Yes & 99 & $(34.4)$ \\
\hline No & 189 & $(65.6)$ \\
\hline
\end{tabular}

TMJ: Temporomandibular joint

(3.1\%) subjects had the maximum score of 60 indicating no impact. When the SC scoring method was used, 21 (7.3\%) subjects had a score of zero and $11(3.8 \%)$ subjects had the maximum score of 12 . The most common negative impact (sometimes, often, or always) was sensitivity of teeth or gums to hot, cold, or sweets.

Mean GOHAI score was 40.9 ( $\mathrm{SD}=10.6$, range: 12 to $60)$. The mean number of negative impacts (sometimes, often, or always) was $6.1(\mathrm{SD}=3.5$, range: 0 to12).

Factor analysis, reliability, and validity

Only one component was extracted. The factor loadings ranged from 0.51 to 0.78 for all items except for item 5 . Cronbach's alpha for the GOHAI was 0.88 , indicating a high degree of internal consistency and homogeneity between the GOHAI items. The item-scale correlation coefficients ranged from 0.53 to 0.77 for all items except item 5, which had a correlation coefficient of 0.27 . The mean values of the individual GOHAI items were close to each other. Test-retest correlation coefficients ranged from 0.41 to 0.75 for all individual items except item 5 , which had a test-retest coefficient of 0.01 . The test-retest correlation for the total add-GOHAI score was 0.72 indicating good stability.

Concurrent validity for the GOHAI was evaluated by examining the correlation between self-perceived general and oral health status and the two GOHAI summary scores (Table 3). Add-GOHAI scores increased with poorer perceived general and oral health. As self-reported general and oral health decreased, SC score (number of negative impacts) increased, indicating poorer health and oral health-related quality of life. Furthermore, people who perceived themselves as needing dental care or those who were not satisfied with their oral health status had a significantly lower mean GOHAI and higher mean SCGOHAI scores, indicating poorer oral health-related quality of life.

As predicted, lower add-GOHAI scores were associated with self-reported TMJ pain, burning mouth sensation, and bad breath, a finding that supports convergent validity (Table 4). Participants who had one or more missing or decayed teeth had lower add-GOHAI scores than those who had no missing or decayed teeth. Those with periodontal disease had lower add-GOHAI scores than those without periodontal disease $(P<0.005)$, supporting construct validity of the add-GOHAI.

Discriminant validity was evaluated by examining the association between GOHAI scores and self-reported bad oral habits which were hypothesized not to be notably associated with oral health-related quality of life and thus have no effect on the GOHAI scores. Table 5 shows that there was no statistically significant difference in GOHAI scores between those who reported bad oral habits and those who did not. Moreover, GOHAI could not discriminate between participants according to the number of filled or crowned teeth.

GOHAI scores were also studied among groups known to have different levels of health. Results were as expected. Respondents who were older than 30 and those with a low level of education had lower add-GOHAI and higher SCGOHAI scores. GOHAI discriminated between the subjects according to tooth brushing; those who regularly brushed their teeth had higher add-GOHAI scores than others (Table 6). 
Table 3 Concurrent validity: Correlation between self-reported general and oral health and the Geriatric Oral Health Assessment Index (GOHAI) scores

\begin{tabular}{lcc}
\hline \multirow{2}{*}{ Item } & \multicolumn{2}{c}{ Pearson's correlation coefficient } \\
\cline { 2 - 3 } & Add-GOHAI & SC-GOHAI \\
\hline Self-perceived general health & -0.30 & 0.31 \\
$(1=$ excellent, $2=$ very good, $3=$ good, $4=$ fair, 5 = poor $)$ & & 0.69 \\
$\begin{array}{l}\text { Self-perceived oral health status } \\
(1=\text { excellent, } 2=\text { very good, } 3=\text { good, } 4 \text { = fair, } 5=\text { poor })\end{array}$ & -0.70 & 0.69 \\
\hline
\end{tabular}

Table 4 Convergent validity: Differences in the average of the Geriatric Oral Health Assessment Index (GOHAI) scores (Add-GOHAI and SC-GOHAI) according to self-reported responses to different health-related questions and objective assessment of oral health

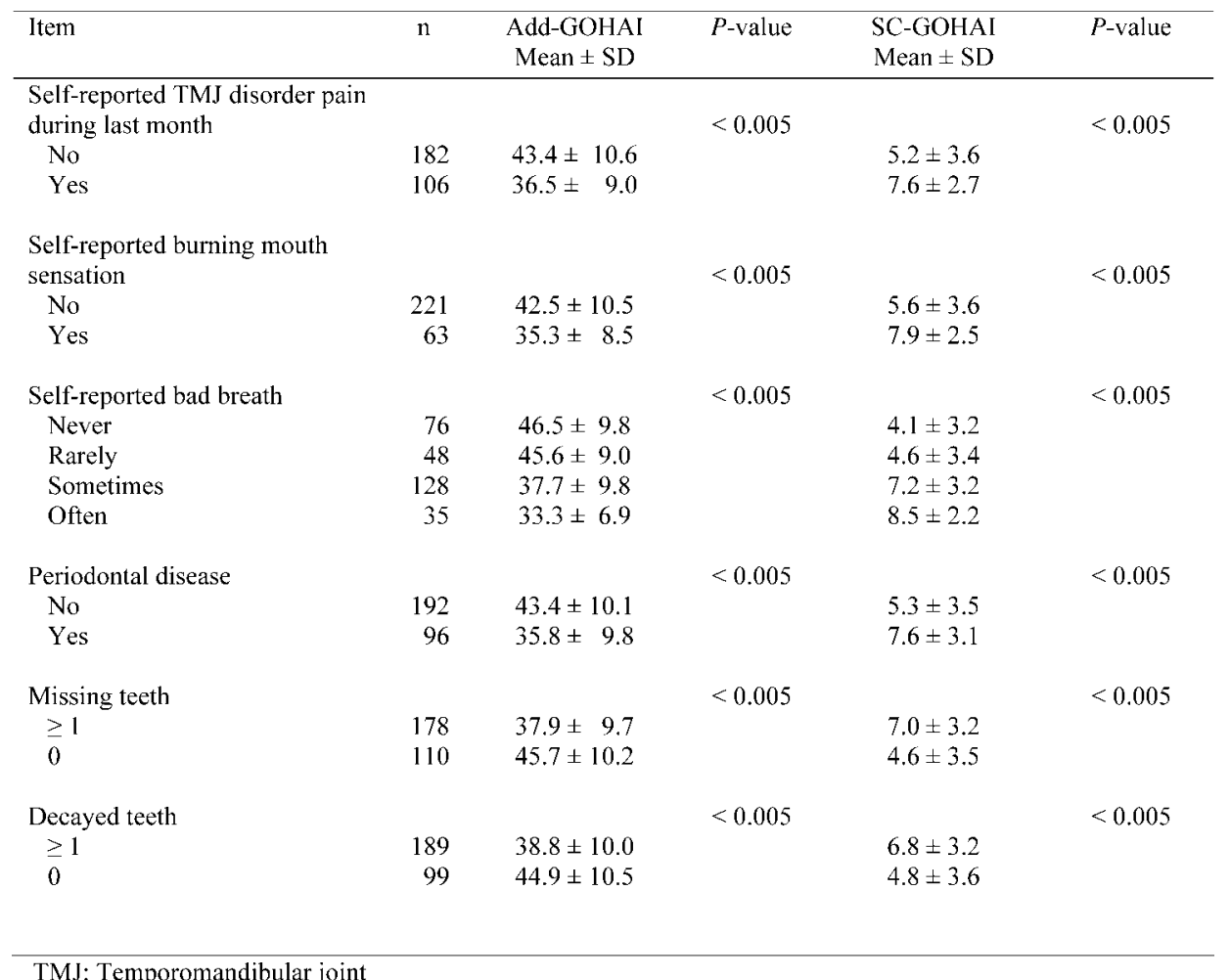

TMJ: Temporomandibular joint

Table 5 Discriminant validity: Differences in the average of the Geriatric Oral Health Assessment Index (GOHAI) scores (Add-GOHAI and SC-GOHAI) according to variables that have no predicted effect on oral health related quality of life

\begin{tabular}{lrcccc}
\hline Item & $\mathrm{n}$ & $\begin{array}{c}\text { Add-GOHAI } \\
\text { Mean } \pm \text { SD }\end{array}$ & $P$-value & $\begin{array}{c}\text { SC-GOHAI* } \\
\text { Mean } \pm \text { SD }\end{array}$ & $P$-value \\
\hline Self-reported bad oral habits & 189 & $41.2 \pm 11.0$ & & $5.9 \pm 3.6$ & 0.174 \\
$\quad$ No & 99 & $40.2 \pm 9.8$ & & $6.5 \pm 3.4$ & \\
$\quad$ Yes & 140 & $40.1 \pm 10.1$ & 0.215 & $6.5 \pm 3.4$ & 0.077 \\
$\begin{array}{l}\text { Filled teeth } \\
\quad \geq 1\end{array}$ & 146 & $41.7 \pm 10.9$ & & $5.7 \pm 3.6$ & \\
$\quad 0$ & 47 & $38.8 \pm 10.4$ & 0.147 & $6.6 \pm 3.1$ & \\
Crowned teeth & 241 & $41.3 \pm 10.6$ & & $6.0 \pm 3.6$ & 0.297 \\
$\geq 1$ & & & & &
\end{tabular}


Table 6 Association between variables with predicted effect on oral health-related quality of life and the GOHAI score

\begin{tabular}{|c|c|c|c|c|c|}
\hline Item & n & $\begin{array}{c}\text { Add-GOHAI } \\
\text { Mean } \pm \text { SD }\end{array}$ & $P$-value & $\begin{array}{l}\text { SC-GOHAI } \\
\text { Mean } \pm \text { SD }\end{array}$ & $P$-value \\
\hline Age (year) & & & 0.005 & & 0.009 \\
\hline$\leq 30$ & 134 & $42.8 \pm 10.4$ & & $5.5 \pm 3.5$ & \\
\hline$>30$ & 142 & $39.2 \pm 10.2$ & & $6.6 \pm 3.3$ & \\
\hline Educational level & & & $<0.005$ & & $<0.005$ \\
\hline Illiterate & 80 & $37.5 \pm 10.0$ & & $7.2 \pm 3.4$ & \\
\hline High school or less & 164 & $41.9 \pm 10.5$ & & $5.7 \pm 3.5$ & \\
\hline More than high school & 44 & $43.3 \pm 10.5$ & & $5.4 \pm 3.4$ & \\
\hline Tooth brushing & & & $<0.005$ & & $<0.005$ \\
\hline$\geq 1 /$ day & 118 & $42.7 \pm 10.8$ & & $5.5 \pm 3.5$ & \\
\hline Not regular & 109 & $40.5 \pm 10.3$ & & $6.2 \pm 3.4$ & \\
\hline Never & 50 & $36.4 \pm 9.0$ & & $7.5 \pm 3.2$ & \\
\hline
\end{tabular}

\section{Discussion}

This study examined the validity and reliability of the GOHAI Arabic version in a selected group of people in north Jordan. The GOHAI, which was originally developed and tested in well-educated, elderly Americans (3) has also been demonstrated suitable in younger, poorly educated populations.

When used among Jordanian people, the Arabic version of the GOHAI showed acceptable validity and reliability. However, it was noticeable that the proportion of subjects reporting discomfort when eating anything (Q5) was lower than that reporting other problems. This item was also atypical in other ways. It had a low item-scale correlation and was poorly correlated with other items. The most likely explanation is that this item was one of three items worded in a positive direction while the remaining items were worded in a negative direction, which might have caused a misunderstanding. The three positively worded items had the lowest item-scale correlations.

Factor analysis extracted only one component. Factor loadings ranged from 0.51 to 0.78 for all items except for item 5, suggesting that the Arabic version of GOHAI demonstrated good internal consistency. Cronbach's alpha coefficient $(0.88)$ was comparable to that obtained from the French version (0.86) (12) and higher than those for versions of the GOHAI in other languages, which varied from 0.74 to $0.81(3,8-10,17)$. Item-scale correlations varied from 0.27 to 0.77 in the Arabic version, compared with 0.28 to 0.61 in the Chinese version (10) and 0.40 to 0.78 in the French version (11).

When 30 subjects retook the questionnaire one week after it was first administered, the test-retest correlation coefficient between the add-GOHAI scores was 0.72 . This finding was similar to that reported for the Malay version (0.72) (9) and lower than that reported for the French version (0.87) (11).
Self-rating of oral health was poor and perception of need for dental treatment was very high, indicating a substantial negative impact of oral conditions among this population. The low socio-economic status of this population may explain the relatively large negative impact of oral conditions on functioning and well-being.

Concurrent validity of the Arabic GOHAI version was comparable to that of the original English GOHAI version (3). The analysis demonstrated the expected associations between the GOHAI score and the reported oral and general health status, perceived need for dental treatment, and self satisfaction with oral health. Lower add-GOHAI scores were associated with poorer perceived oral and general health, need for dental care, and low satisfaction with oral health.

Regarding convergent validity, this study supported others (2) in showing that people with TMJ pain, burning mouth sensation, or self reported bad breath had lower addGOHAI scores than those who did not have these symptoms. Bad oral habits did not have any significant effect on the GOHAI score. However, education and employment were important factors influencing the GOHAI score, as reported previously $(7,17)$.

This study found significant relationships between the GOHAI and certain clinical measures including number of missing teeth, number of decayed teeth, and periodontal status. However, associations between the GOHAI score and the number of filled or crowned teeth were not significant. Missing and decayed teeth affect eating, esthetics, and speech, and dental decay may cause pain and discomfort. Periodontal diseases cause tooth mobility, tooth loss, limiting the ability to eat comfortably and resulting in esthetic and speech problems. On the other hand, fillings and crowns are designed to restore as much of the lost function and esthetics as possible, accounting for the fact that those with filled and crowned teeth did 
not score significantly worse on the GOHAI than others.

In conclusion, the Arabic translation of the GOHAI demonstrated acceptable validity and reliability when used for people in north Jordan. It could therefore be a valuable instrument for measuring oral health-related quality of life for people in this region. Further research is needed to examine the stability of the GOHAI over different periods of time and to examine it as a tool to evaluate dental treatment outcomes in the Jordanian population.

\section{References}

1. Fitzpatrick R, Fletcher A, Gore S, Jones D, Spiegelhalter D, Cox D (1992) Quality of life measures in health care. I: Applications and issues in assessment. BMJ 305, 1074-1077

2. Slade GD, Spencer AJ (1994) Development and evaluation of the Oral Health Impact Profile. Community Dent Health 11, 3-11

3. Atchison KA, Dolan TA (1990) Development of the Geriatric Oral Health Assessment Index. J Dent Educ 54, 680-687

4. Strauss RP, Hunt RJ (1993) Understanding the value of teeth to older adults: influences on the quality of life. J Am Dent Assoc 124, 105-110

5. McGrath C, Bedi R (2003) Measuring the impact of oral health on quality of life in Britain using OHQoL-UK(W). J Public Health Dent 63, 73-77

6. Leao A, Sheiham A (1996) The development of a socio-dental measure of dental impacts on daily living. Community Dent Health 13, 22-26

7. Atchison KA (1997). The General Oral Health Assessment Index. In Measuring Oral Health and Quality of Life, Slade GD ed, Chapel Hill, 79-80

8. Hägglin C, Berggren U, Lundgren J (2005) A
Swedish version of the GOHAI index. Psychometric properties and validation. Swed Dent J 29, 113124

9. Othman WN, Muttalib KA, Bakri R, Doss JG, Jaafar N, Salleh NC, Chen S (2006) Validation of the Geriatric Oral Health Assessment Index (GOHAI) in the Malay language. J Public Health Dent 66, 199204

10. Wong MC, Liu JK, Lo EC (2002) Translation and validation of the Chinese version of GOHAI. J Public Health Dent 62, 78-83

11. Tubert-Jeannin S, Riordan PJ, Morel-Papernot A, Porcheray S, Saby-Collet S (2003) Validation of an oral health quality of life index (GOHAI) in France. Community Dent Oral Epidemiol 31, 275-284

12. Guillemin F, Bombardier C, Beaton D (1993) Crosscultural adaptation of health-related quality of life measures: literature review and proposed guidelines. J Clin Epidemiol 46, 1417-1432

13. Allison P, Locker D, Jokovic A, Slade G (1999) A cross-cultural study of oral health values. J Dent Res 78, 643-649

14. Corless IB, Nicholas PK, Nokes KM (2001) Issues in cross-cultural quality-of-life research. J Nurs Scholarsh 33, 15-20

15. Cronbach LJ (1951) Coefficient alpha and the internal structure of tests. Psychometrika 16, 297 334

16. John MT, Patrick DL, Slade GD (2002) The German version of the Oral Health Impact Profile/translation and psychometric properties. Eur J Oral Sci 110, 425433

17. Allen PF (2003) Assessment of oral health related quality of life. Health Qual Life Outcomes 1, 40 